\title{
BMJ Open Screening and receiving information for intimate partner violence in healthcare settings: a cross-sectional study of Arab and Jewish women of childbearing age in Israel
}

Nihaya Daoud, ${ }^{1}$ Alexandra Berger-Polsky, ${ }^{1}$ Ruslan Sergienko, ${ }^{1}$ Patricia O'Campo, ${ }^{2}$ Rebecca Leff, ${ }^{3}$ Ilana Shoham-Vardi ${ }^{1}$

To cite: Daoud N, BergerPolsky A, Sergienko R, et al. Screening and receiving information for intimate partner violence in healthcare settings: a cross-sectional study of Arab and Jewish women of childbearing age in Israel. BMJ Open 2019;9:e022996. doi:10.1136/ bmjopen-2018-022996

- Prepublication history and additional material for this paper is available online. To view these files, please visit the journal online (http://dx.doi.org/10. 1136/bmjopen-2018-022996).

Received 17 March 2018 Revised 21 December 2018 Accepted 8 January 2019

D Check for updates

(C) Author(s) (or their employer(s)) 2019. Re-use permitted under CC BY-NC. No commercial re-use. See rights and permissions. Published by BMJ.

${ }^{1}$ Department of Public Health, Faculty of Health Sciences, BenGurion University of the Negev, Beer -Sheva, Israel

${ }^{2}$ Centre for Urban Health Solutions, St.Michael's Hospital, Toronto, Ontario, Canada ${ }^{3}$ School of Medicine, Faculty of Health Sciences, Ben-Gurion University of the Negev, Beer -Sheva, Israel

Correspondence to

Dr Nihaya Daoud;

daoud@bgu.ac.il

\section{ABSTRACT}

Objectives We studied the proportion of women who have ever been screened (ES) for intimate partner violence (IPV) in a healthcare setting, received information (RI) about relevant services, or both, and explored disparities in screening and information provision by ethnicity and other characteristics.

Design In 2014-2015, we undertook a cross-sectional study, conducting interviews using a structured questionnaire among a stratified sample of $1401 \mathrm{Arab}$ and Jewish women in Israel.

Setting A sample of 63 maternal and child health clinics (MCH) clinics in four geographical districts.

Participants Women aged 16-48years, pregnant or up to 6 months after childbirth.

Primary and secondary outcome measures We used multivariable generalised estimating equation analysis to determine characteristics of women who were ES (Has anyone at the healthcare services (HCS) ever asked you whether you have experienced IPV?); RI (Have you ever received information about what to do if you experience IPV?); and both (ES\&RI).

Results Less than half of participants (48.8\%) reported ES; $50.5 \% \mathrm{Rl}$; and $30 \%$ were both ES\&RI. Having experienced any IPV was not associated with ES or ES\&RI but was associated with $\mathrm{RI}$ in an unexpected direction. Women at higher risk for IPV (Arab minority women, lower education, unmarried) were less likely to report being ES, RI or both. The OR and 95\% Cl for not ER\&RI were: 1.58 (1.00 to 2.49) among Arab compared with Jewish women; 1.95 (1.42 to 2.66) among low education versus academic education women; 1.34 (1.03 to 1.73) among not working versus working. ES, RI and both differ across districts.

Conclusions While Israel mandates screening and providing information regarding IPV for women visiting the HCS, we found inequalities, suggesting inconsistencies in policy implementation and missed opportunities to detect IPV. To increase IPV screening and information provision, the ministry of health should circulate clarification and provide support to healthcare providers to conduct these activities.

\section{Strengths and limitations of this study}

- Our study is the first that we know of to quantify implementation of intimate partner violence (IPV) screening and information provision, with attention to ethnicity and other characteristics of women who do or do not receive these services.

- We found inequalities in IPV screening and information provision, suggesting inconsistent implementation of the screening policy by healthcare providers.

- One limitation is that having ever been screened for, or received information on IPV were self-reported, thus recall bias could have occurred.

- Another limitation is that our sample was composed of women visiting Ministry of Health Maternal and Child Health clinics, women visiting other clinics might have different experiences.

\section{INTRODUCTION}

Affecting more than one-third of women globally, ${ }^{1}$ intimate partner violence (IPV) has been shown to have a major effect on women's physical and mental health. ${ }^{2}$ Women who experience IPV tend to use more healthcare services $(\mathrm{HCS})^{34}$ and are likely to disclose abuse for the first time to a healthcare provider (HCP), ${ }^{5}$ as the latter are often seen as trusted professionals. ${ }^{6}$ Therefore, HCPs can play a critical role in detection of IPV within HCS if they ask women about IPV. ${ }^{7}$ While the effectiveness of IPV screening has yet to be fully demonstrated in research, ${ }^{8}$ studies show that it can encourage women to disclose IPV which might increase detection. ${ }^{7} 9$ Just as importantly, IPV screening within HCS can become an occasion to provide victims with information about and referrals to supportive services, ${ }^{79}$ as well as to consult with them on how to cope. 710 
A Cochrane review ${ }^{8}$ distinguishes between different approaches to identifying women exposed to IPV in HCS contexts; universal screening prescribes screening for all women interacting with HCS in all locations via standardised questions and procedures, regardless of 'symptoms' or risk factors; selective or targeted screening focuses on women with specific characteristics, such as when pregnant or seeking to terminate a pregnancy, and screens them using the same question; routine inquiry asks all women about IPV, but using varying methods or questions according to HCPs and particular women's situations. A case-finding is different from screening and it asks women about IPV if they present with symptoms or characteristics of IPV exposure. Proponents of universal screening argue that the severity of the burden of suffering from IPV necessitates a universal protocol. ${ }^{11} 12$ They note that screening for IPV within HCS is associated with minimal risk, discomfort and emotional distress and has been shown to increase detection, reduce IPV and improve the health of screened women. ${ }^{13}$ However, some reviews cast doubt on this view, citing a lack of evidence regarding the benefits of universal screening and suggest a casefinding approach to identifying IPV. ${ }^{8}{ }^{14}$ Regardless, since the late 1990s, many health-professional associations have published clinician guidelines on how to identify and respond to women who have been abused, ${ }^{15} 16$ and health professionals are now increasingly required to undertake screening in accordance with national health policies. For example, in 2013, the Preventive Services Task Force in the USA recommended universal IPV screening of all women of childbearing age and referral for intervention services for women who screen positive. ${ }^{17}$ Conversely, some European countries (eg, the UK and Sweden) have enacted a case finding approach to detect women with IPV. 81819

However, even in countries where medical organisations have recommended universal screening for IPV within HCS, ${ }^{11} 16182021$ studies show that HCPs do not necessarily carry out this mandate. ${ }^{182223}$ In clinic-based studies in the USA, the proportion of screening at least once by physicians was $11 \%-39 \% .{ }^{24}$ This low proportion appears to be due to different barriers; clinicians may not feel confident about screening, not know what questions to ask or how to respond if a woman says she is being abused, may feel there is not enough time to screen or may see other issues as taking precedence. ${ }^{92}$ Barriers can also come from women, who may not trust the provider enough to disclose this sensitive information. ${ }^{925}$ This may relate to HPC attitudes towards IPV. For example, Gutmanis $e t a l^{23}$ identified misconceptions about IPV among HCPs, such as: 'domestic violence is rare,' and 'domestic violence is a private matter that should be resolved without outside intervention. ${ }^{23}$ Nursing staff, while generally expressing more favourable views toward screening than physicians, have also been shown to face internal barriers to screening, especially a fear of offending patients during questioning. ${ }^{26}$ While these seem to be individual level barriers among HCPs, Gracia-Moreno et al identified health system-level barriers, including the interests of government leadership and the political will to implement a comprehensive health-system approach, as affecting whether or not HCPs carry out their role in identifying and helping women victims of IPV and sexual violence. ${ }^{27}$

Meanwhile, research shows women generally support universal IPV screening, ${ }^{28}$ and a meta-analysis of 25 studies showed they want HCPs to be non-judgemental, non-directive and understanding of the complexity of partner violence ${ }^{619}$ However, other studies suggest that minority women and those with low socioeconomic status, who experience higher prevalence of IPV, ${ }^{29-31}$ might be less likely to be screened by HCPs or RI about IPV services. One study in Hawaii showed that, compared with White women, women from minority groups receive less counselling on IPV during prenatal care. ${ }^{32}$ This suggests that minority women are screened for IPV less often. However, none of the studies we know of shows such disparities, and few studies have been conducted on disparities in IPV screening and information provision among women at risk for IPV.

\section{IPV screening in HCS settings in Israel}

Israel is an apt setting for investigating implementation of universal IPV screening due to its ethnic makeup, with majority-Jewish and minority indigenous-Arab populations highly stratified by class, socioeconomic status and geography. This stratification might differentially affect the likelihood that women will feel at ease disclosing IPV in a healthcare setting. ${ }^{33}$ A recent study on IPV found that Arab minority women and low-income women in Israel are more likely to report IPV compared with Jewish women. Risk factors for IPV among Arab women included younger age, higher level of religiosity and living in urban areas. ${ }^{33}$ To respond to domestic violence, in 2003 the Israeli Ministry of Health (MOH) issued Director General Circular

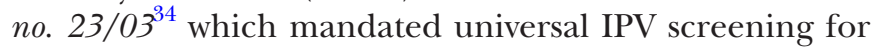
all women who visit HCSs. Yet, the Circular's approach to screening is inconclusive. ${ }^{34}$ It states that 'all women who present at the HCS should be asked about IPV' which echoes universal screening or routine inquiry (depending on the screening tool), but also notes that 'specific emphasis should be on women who present with symptoms,' implying a case finding (not screening). It goes on to say that all pregnant women who visit MCH clinics should be asked about IPV, suggesting targeted or selective screening. ${ }^{34}$ This inconclusive wording contrasts with the conclusive approaches delineated in the above-mentioned Cochrane review definitions. ${ }^{8}$ The Circular thus fails to offer clear direction for IPV screening. This might be a source of confusion for HCPs.

Indeed, a 2010 report by the MOH showed that HCPs hesitate to implement the Circular's recommendations. ${ }^{35}$ The report, based on in-depth interviews with nurses and physicians, identified different barriers to IPV screening and found that nurses are more likely to ask about IPV than physicians, but that both are reluctant to screen. ${ }^{35}$ Few studies on the practice of IPV screening in Israel 
are available, and most have used small hospital-based samples. For example, Ben Natan et al. ${ }^{36}$ studied a sample of 100 physicians and nurses from obstetrics and gynaecology departments in a Central Israel hospital. They found that the most cited reason for failure to screen for IPV among HCPs was that they are embarrassed by the intimate nature of screening, including questioning patients. ${ }^{36}$ And a previous nationwide study conducted in 2000 showed that only $3 \%$ of women were asked about domestic violence (not IPV) by a physician at primary care clinics. ${ }^{37}$

Despite these findings, little data yet exist to assess implementation of IPV screening or provision of information about related services across Israeli HCSs. Our study aims to examine the proportion of women who have ever been screened (ES) for IPV within HCSs, who have received information (RI) about IPV services, and both; and to determine characteristics, including ethnicity, associated with ES, RI and both (ES \& RI).

\section{Methods}

Study design and sampling

Data were obtained from our cross-sectional study on 'Family Relations, Violence and Health'29 (https:// www.ncbi.nlm.nih.gov/pubmed/29294734), conducted between October 2014 and October 2015 with approval by the Public Health Division of the Israeli MOH. Data collection followed the WHO ethical recommendations on safety of women victims of abuse. ${ }^{38}$ A more detailed description of sampling can be found elsewhere, ${ }^{29}$ but briefly, we selected Maternal and Child Health (MCH) clinics via a stratified sampling procedure based on Israel's main regions (South, Center, North and Haifa), the proportion of births in each region or district, and the ethnic composition (Arabs vs Jews) of the population of women in the region. $\mathrm{MCH}$ clinics are located in neighbourhoods and provide prenatal care, well-child follow-up and required immunisations for different population groups. In this manner, we selected $63 \mathrm{MCH}$ clinics: 33 in Jewish localities, 21 in Arab localities and 9 in mixed localities.

\section{Participants and data collection}

Participants were recruited at the $63 \mathrm{MCH}$ clinics that were sampled for the study. One month before data collection, the study coordinators distributed leaflets at these clinics with information about the study. Trained female interviewers asked eligible mothers (pregnant, or 6 weeks to 6 months after childbirth) to participate in the study. Women who agreed to participate were invited into a separate room at the clinics, where they were interviewed face to face using a structured questionnaire in the women's preferred language (Arabic or Hebrew) after signing an informed consent form. If a participant was detected as having experienced IPV, the interviewer and the study team encouraged her to talk with the MCH nurse who could refer her to a social worker in the community's social services office. In addition, all study participants received written contact information on community services that support women who are victims of violence. A total of 1401 women were interviewed (436 Arab and 965 Jewish), with a response rate of $76 \%$ among Arab women, $73 \%$ among Jewish women. Our sample size was calculated using Winpepi software V.11.65. Our calculation was based on results of a study conducted in Northern Israel by Fisher et al. ${ }^{39}$ They found $8 \%$ prevalence of IPV in the total sample, with $26 \%$ of Arab women reporting IPV compared with $16 \%$ of Jewish women. To detect a $10 \%$ difference of IPV between these two ethnic groups with a significance level of $5 \%$, power of $90 \%$ and prevalence of IPV 8\%, we needed a total sample of 1156 women with a cluster size of 25 participants per cluster (MCH clinic) and 46 clusters (MCH clinics). This was after multiplying by 0.005 for the Inter Cluster Correlation effect, as recommended in cluster design health studies. ${ }^{40}$ We added $20 \%$ to the sample size (as originally this was a cohort design study and we assumed women would be lost for follow-up). Therefore, the final sample size was 1401 women interviewed at 63 clinics, as we ended up interviewing 14 more women than the required sample of 1387 women.

\section{Measures}

\section{Dependent variables}

ES for IPV: a yes/no question: 'Has anyone at a HCS ever asked you whether you have experienced IPV?'

$R I$ about IPV services: a yes/no question: 'Have you ever received information about what to do in case you experience IPV?"; this question was asked regardless of responses to the preceding question.

An index variable of ESE RI: We created an index variable that included positive answers to the previous two questions versus all other answers.

\section{Independent Variables}

Any IPV: any positive answer to a list of 10 questions about acts of violence perpetrated by a participant's intimate partner at any time. ${ }^{29} 41$ These acts include physical violence, psychological violence, social control and economic violence. For example, 'Your partner has hit you, kicked you, pushed you or thrown things at you?'; 'Does your partner try to isolate you from your family and friends?' and 'Are you fearful of drastic changes in your partner's mood?.', Our list of questions was based on those used in the Preventive Services Task Force Family screening tool on IPV, ${ }^{41}$ and on questions that are used for IPV screening in some MCH clinics in Israel. For information about the tool used to assess IPV, please see Daoud et al. . $^{33}$

Types of IPV: resulted from factor analysis for the above 10 acts of IPV, and were categorised as: physical or sexual violence (eg, beating, slapping and kicking; forced sex); emotional or verbal violence (eg, threats of harm, constant humiliation, insults); and social or economic violence ${ }^{33}$ (controlling behaviours, such as isolating a woman from her family and friends, monitoring her movements and 
restricting access to financial resources including employment, education or medical care).

Ethnicity: self-determined as (1) Arab or (2) Jewish.

Immigrant Status: born in Israel or another country. Age: 16-24, 25-34 and 35-48 years old.

Marital status: (1) married, and (2) not married, including single, divorced, separated, not-cohabitating or other.

Women's status during the interview: a composite variable based on answers to three questions: 'Are you currently pregnant?' (yes/no), 'Do you have children?' (yes/no) and 'How many children do you have?' We categorised answers as follows: (1) pregnant with no children, (2) pregnant with children, (3) not pregnant with 1-2 children and (4) not pregnant with three children or more.

Women's education and husband's education: (1) high school or less, (2) postsecondary education and (3) university education (Bachelor's, Master's or Doctorate).

Employment status for women and husbands: a yes/no question about current work outside the household by participants and their partners.

Family source of income: (1) work only, (2) social allowances only or (3) other source which included any combination of work and social allowances, and work and other resources, such as a grant, family support, land or other source of income.

Religiosity: (1) religious or very religious, (2) traditional, (3) not religious. ${ }^{42} 43$

$\mathrm{MOH}$ district: based on the country's geographic areas. We categorised these into four districts: We named these districts as A, B, C and D to keep the information about the district's performance anonymous.

\section{Statistical analysis}

We calculated the proportion (\%) of our main variables (ES, RI and the index variable of ES\&RI) for the total sample of women, and examined women's characteristics across these variables using the $\chi^{2}$ test. Next, we examined univariate associations between IPV; types of IPV; and ES, RI and the ES\&RI index variable. Since Arab and Jewish women differed in most of the independent and dependent variables, we examined interactions between ethnicity and each of the associations between independent variables and ER, RI and the index variable (ES\&RI). The interaction with the $\mathrm{MOH}$ district variable was positive. Therefore, we decided to conduct our multivariate analysis for each ethnic group (Arab and Jewish) in addition to the multivariable analysis for the total sample. We used generalised estimating equation (GEE) analysis in the multivariable analysis to adjust for the $\mathrm{MCH}$ clinic cluster effect, while adjusting for significant $(p<0.05)$ independent variables that were associated with ES, RI and the ES\&RI index variable in the univariate analysis. Three multivariable analysis models were fitted for each study group in order to examine characteristics of women who received the services we studied: ES, RI and ES\&RI.

The correlation between the study independent variables was lower than our threshold of $\mathrm{R}>0.4$, and we did not exclude any of these variables from the multivariable analysis, as multicollinearity was not likely (see online supplementary appendix 1 ).

\section{Patient and public involvement}

Patients and the public were not involved in this research.

\section{Results}

Less than half of the women reported ES for IPV (48.4\%), close to half $(50.5 \%)$ RI about services for coping with IPV and only $30.4 \%$ reported both ES\&RI (table 1).

Table 1 also presents characteristics of women who received each of these services out of the total sample of participants. Notably, experiencing any IPV, physical and emotional IPV was not associated with having ES for IPV. However, women who reported less social IPV were more likely to report ES. In addition, reporting ES was higher among Jewish women; married women; those not pregnant with three children or more; women with postsecondary education or an academic degree; employed women with main source of family income from work; religious or very religious women; and those living in districts $\mathrm{C}$ and D. Age, immigrant status and partner employment were not associated with reporting ES for IPV.

Reporting RI on services for coping with IPV was more likely among women who reported not experiencing any IPV, or emotional or social IPV. Physical IPV was not associated with RI. However, reporting RI was higher among Jewish women; older women; women not pregnant; with 1-2 children; women with academic education; employed women, and women with employed partners; women with an income source from work and other sources; women who are not religious; and women who live in district B. RI was not associated with immigrant or marital status (table 1).

Women who reported both ES \& RI were more likely to be Jewish, older, not pregnant during the interview, with children, with higher education, employed and with family income from other sources. However, the variables of any IPV, types of IPV, immigrant status, marital status, partner employment, religiosity and $\mathrm{MOH}$ district were not associated with reporting both services (ES\&RI).

For the multivariable analysis, we considered variables that were significantly associated in the bivariate analysis with each of our outcome variables of ES, RI and both (ES\&RI). Since we found significant interactions of ethnicity (Jewish vs Arab) and the associations between the $\mathrm{MOH}$ district variable and ES, RI and ES\&RI, we conducted GEE multivariable analysis for each of these dependent variables (ES, RI and both) for the total sample of women and then separately for each ethnic group. The results of the multivariable analysis are presented in tables 2-4.

Results of the GEE for having been ES for IPV among the total sample (table 2) show that the OR of not having been ES was higher among women at higher risk for IPV. This includes Arab compared with Jewish women (OR, $95 \%$ CI $1.98,1.16$ to 3.36 ); and women who experience 
Table 1 Univariate association for ever being screened (ES) for intimate partner violence (IPV), receiving information (RI) about IPV services, and both, among women in the study sample in Israel, 2014-2015

\begin{tabular}{|c|c|c|c|c|c|c|c|}
\hline \multirow[b]{2}{*}{ Women's characteristics } & \multirow{2}{*}{$\begin{array}{l}\text { Total sample } \\
\mathrm{n}=1401 \\
\mathrm{~N}(\%)\end{array}$} & \multicolumn{2}{|l|}{ ES for IPV } & \multicolumn{2}{|c|}{ RI about IPV services } & \multicolumn{2}{|l|}{ ES\&RI } \\
\hline & & N (\%) & $P$ value & N (\%) & $P$ value & $\mathbf{N}(\%)$ & $P$ value \\
\hline Total & & $675(48.4)$ & & $708(50.5)$ & & $425(30.4)$ & \\
\hline Any IPV & & & 0.250 & & 0.011 & & 0.134 \\
\hline Yes & $843(39.8)$ & $259(46.5)$ & & $259(46.4)$ & & $157(28.1)$ & \\
\hline No & $558(60.2)$ & $416(49.6)$ & & $449(53.4)$ & & 268 (31.9) & \\
\hline Physical IPV & & & 0.805 & & 0.925 & & 0.677 \\
\hline No & $1333(95.1)$ & $643(48.4)$ & & $674(50.6)$ & & $407(30.6)$ & \\
\hline Yes & $64(4.6)$ & $32(50.0)$ & & $32(50.0)$ & & $18(28.1)$ & \\
\hline Emotional IPV & & & 0.397 & & 0.005 & & 0.104 \\
\hline No & $997(71.4)$ & $489(49.2)$ & & $527(52.9)$ & & $316(31.8)$ & \\
\hline Yes & 399 (28.6) & $186(46.7)$ & & $178(44.6)$ & & 109 (27.3) & \\
\hline Social IPV & & & 0.038 & & 0.007 & & 0.115 \\
\hline No & $1033(73.7)$ & $516(50.1)$ & & $544(52.7)$ & & $326(31.6)$ & \\
\hline Yes & $364(26.1)$ & $159(43.8)$ & & $162(44.5)$ & & 99 (27.2) & \\
\hline Ethnicity & & & $<0.001$ & & $<0.001$ & & $<0.001$ \\
\hline Arab & $434(31.1)$ & $162(37.3)$ & & $166(38.1)$ & & $89(20.4)$ & \\
\hline Jewish & 965 (68.9) & $513(53.4)$ & & $542(56.3)$ & & $336(34.9)$ & \\
\hline Age & & & 0.096 & & 0.004 & & 0.010 \\
\hline $16-24$ & $247(17.6)$ & $104(42.4)$ & & $101(40.9)$ & & $56(22.8)$ & \\
\hline $25-34$ & $844(60.3)$ & $413(49.1)$ & & $444(52.7)$ & & $263(31.2)$ & \\
\hline $35-48$ & $309(22.1)$ & $158(51.3)$ & & $162(52.4)$ & & $106(34.3)$ & \\
\hline Immigrant & & & 0.954 & & 0.234 & & 0.588 \\
\hline Not immigrant & $1133(81.4)$ & $547(48.4)$ & & $565(50.0)$ & & $340(30.1)$ & \\
\hline Immigrant & $259(18.6)$ & $124(48.2)$ & & $140(54.1)$ & & $82(31.8)$ & \\
\hline Marital status & & & 0.018 & & 0.129 & & 0.237 \\
\hline Married & $1329(95.2)$ & $650(49.1)$ & & $666(50.2)$ & & $407(30.7)$ & \\
\hline Other & $67(4.8)$ & 23 (34.3) & & $40(59.7)$ & & $16(23.9)$ & \\
\hline Women's status during interview & & & $<0.001$ & & $<0.001$ & & $<0.001$ \\
\hline $\begin{array}{l}\text { Not pregnant with } \\
3+\text { children }\end{array}$ & $390(28.0)$ & $204(52.6)$ & & $194(49.7)$ & & $136(34.9)$ & \\
\hline $\begin{array}{l}\text { Not pregnant with 1-2 } \\
\text { children }\end{array}$ & $737(52.9)$ & $376(51.1)$ & & $412(55.9)$ & & $234(31.8)$ & \\
\hline Pregnant with children & $187(13.4)$ & $70(37.8)$ & & 62 (33.3) & & $38(20.4)$ & \\
\hline Pregnant without children & $80(5.7)$ & $21(26.3)$ & & $36(45.0)$ & & $14(17.5)$ & \\
\hline Education (woman) & & & $<0.001$ & & $<0.001$ & & $<0.001$ \\
\hline High school or less & $537(38.3)$ & 204 (38.2) & & $211(39.3)$ & & $111(20.7)$ & \\
\hline Postsecondary or college & 251 (17.9) & $134(53.6)$ & & $126(50.6)$ & & $82(32.8)$ & \\
\hline Bachelor degree or above & $613(43.8)$ & $337(55.2)$ & & $371(60.5)$ & & $232(37.8)$ & \\
\hline Employment (woman) & & & $<0.001$ & & $<0.001$ & & $<0.001$ \\
\hline Yes & $781(56.6)$ & $414(53.0)$ & & $445(56.8)$ & & $279(35.7)$ & \\
\hline No & $59.8(43.4)$ & $252(42.1)$ & & $257(42.8)$ & & $141(23.5)$ & \\
\hline Employment (partner) & & & 0.791 & & 0.005 & & 0.229 \\
\hline Yes & $1222(88.0)$ & $592(48.7)$ & & $633(51.9)$ & & $379(31.1)$ & \\
\hline No & 166 (12.0) & 79 (47.6) & & 67 (40.0) & & 44 (26.5) & \\
\hline
\end{tabular}


Table 1 Continued

\begin{tabular}{|c|c|c|c|c|c|c|c|}
\hline \multirow[b]{2}{*}{ Women's characteristics } & \multirow{2}{*}{$\begin{array}{l}\text { Total sample } \\
\mathrm{n}=1401\end{array}$} & \multicolumn{2}{|l|}{ ES for IPV } & \multicolumn{2}{|c|}{ RI about IPV services } & \multicolumn{2}{|l|}{ ES\&RI } \\
\hline & & N (\%) & $P$ value & $\mathbf{N}(\%)$ & $P$ value & $\mathbf{N}(\%)$ & $P$ value \\
\hline Household income source & & & 0.001 & & $<0.001$ & & 0.005 \\
\hline Work only & $982(70.1)$ & $466(47.6)$ & & $493(50.3)$ & & $290(29.6)$ & \\
\hline Social allowances only & $79(5.6)$ & 25 (31.6) & & 25 (31.6) & & $14(17.7)$ & \\
\hline Work and other source & 340 (24.3) & $184(54.4)$ & & $190(56.0)$ & & $121(35.7)$ & \\
\hline Religiosity & & & 0.002 & & 0.041 & & 0.604 \\
\hline Not religious & $440(31.5)$ & $220(50.1)$ & & $244(55.6)$ & & $135(30.7)$ & \\
\hline Traditional & $608(43.5)$ & $262(43.4)$ & & $298(49.0)$ & & 177 (29.2) & \\
\hline Religious or very religious & $351(25.1)$ & $193(55.1)$ & & $166(47.4)$ & & $113(32.3)$ & \\
\hline $\mathrm{MOH}$ district & & & 0.001 & & 0.019 & & 0.344 \\
\hline A & $358(25.7)$ & $160(45.1)$ & & $166(46.5)$ & & $113(31.7)$ & \\
\hline B & $212(15.2)$ & $82(38.7)$ & & $127(59.9)$ & & $54(25.5)$ & \\
\hline C & $334(24.0)$ & $183(54.8)$ & & $165(49.4)$ & & $102(30.5)$ & \\
\hline D & 485 (35.0) & $248(51.1)$ & & $248(51.0)$ & & 156 (32.1) & \\
\hline
\end{tabular}

$\mathrm{MOH}$, Ministry of Health.

IPV compared with women who do not experience IPV (OR, 95\% CI 1.30, 1.01 to 1.67$)$. Unmarried women compared with married were almost twice as likely to report not having ES (OR, 95\% CI 2.07,1.23 to 3.27). Women with lower education (high school or less) had a higher likelihood of reporting not having ES compared with women with higher education (OR, 95\% CI 1.75, 1.29 to 2.38$)$.

Among Arab minority women, not having ES was reported more often among women who were not pregnant, with 1-2 children at the time of the interview, compared with those not pregnant with three children or more (OR, 95\% CI 1.61, 1.04 to 2.47). Meanwhile, traditional Arab women were less likely to report not having ES for IPV compared with non-religious Arab women (OR, $95 \%$ CI $0.56,0.36$ to 0.89 ). In addition, women in district $\mathrm{C}$ were less likely to report not having ES compared with women in district A (OR, 95\% CI 0.14, 0.06 to 0.32 ).

For Jewish women, not having ES was reported more often by: unmarried compared with married women (OR, 95\% CI 2.02, 1.20 to 3.40); those with lower education compared with higher or academic education (OR, 95\% CI 1.96, 1.40 to 2.76); and women living in district B compared with district A (OR, 95\% CI 2.08, 1.20 to 3.58). Religious Jewish women were less likely to report not having ES for IPV compared with non-religious women (OR, 95\% CI $0.66,0.49$ to 0.90 ).

Regarding RI on services for coping with IPV, among women in the total sample (table 3) we found that not RI was higher among: Arab compared with Jewish women (OR, 95\% CI 1.79, 1.24 to 2.56); pregnant women with children compared with women who were not pregnant with three children or more at the time of the interview (OR, 95\% CI 1.69, 1.02 to 2.78); women with high school education or less compared with women with academic education (OR, 95\% CI 1.82, 1.37 to 2.34); and women with postsecondary education compared with women with academic-level education (OR, 95\% CI 1.31, 1.04 to 1.66). Women living in district $\mathrm{C}$ and district $\mathrm{B}$ were less likely to report not RI compared with women living in district A (OR, $95 \%$ CI $0.70,0.52$ to 0.93 and OR, $95 \%$ CI 0.66 , 0.42 to 1.04 , respectively).

Among Arab women, not reporting RI was higher among pregnant women with children compared with women who were not pregnant with three children or more (OR, 95\% CI 2.22, 1.17 to 4.22), and among women with lower education compared with higher education (OR, 95\% CI 2.21, 1.31 to 3.72). Women living in district $\mathrm{C}$ compared with district A were less likely to report not RI (OR, 95\% CI $0.45,0.23$ to 0.88 ).

As for Jewish participants, compared with younger women, older women were less likely to report not RI (OR, 95\% CI $0.59,0.39$ to 0.88 ). However, women with lower education compared with academic educated women were more likely to report not RI (OR, 95\% $\mathrm{CI}=1.57,1.11$ to 2.21 ). Also, women living in the district D compared with women in district A were more likely to report not RI (OR, 95\% CI 1.51,1.17 to 1.95 ).

The multivariable results for participants reporting having both ES\&RI are presented in table 4. For the total sample, not reporting both services (ES\&RI) was nearly one and a half times more likely among Arab compared with Jewish women (OR, 95\% CI 1.58,1.00 to 2.49), almost two times more likely in women with high school education or less compared with those with academic education (OR, 95\% CI 1.95, 1.42 to 2.66), unemployed compared with employed women (OR, 95\% CI 1.34, 1.03 to 1.73 ) and women living in district $\mathrm{B}$ compared with district A 


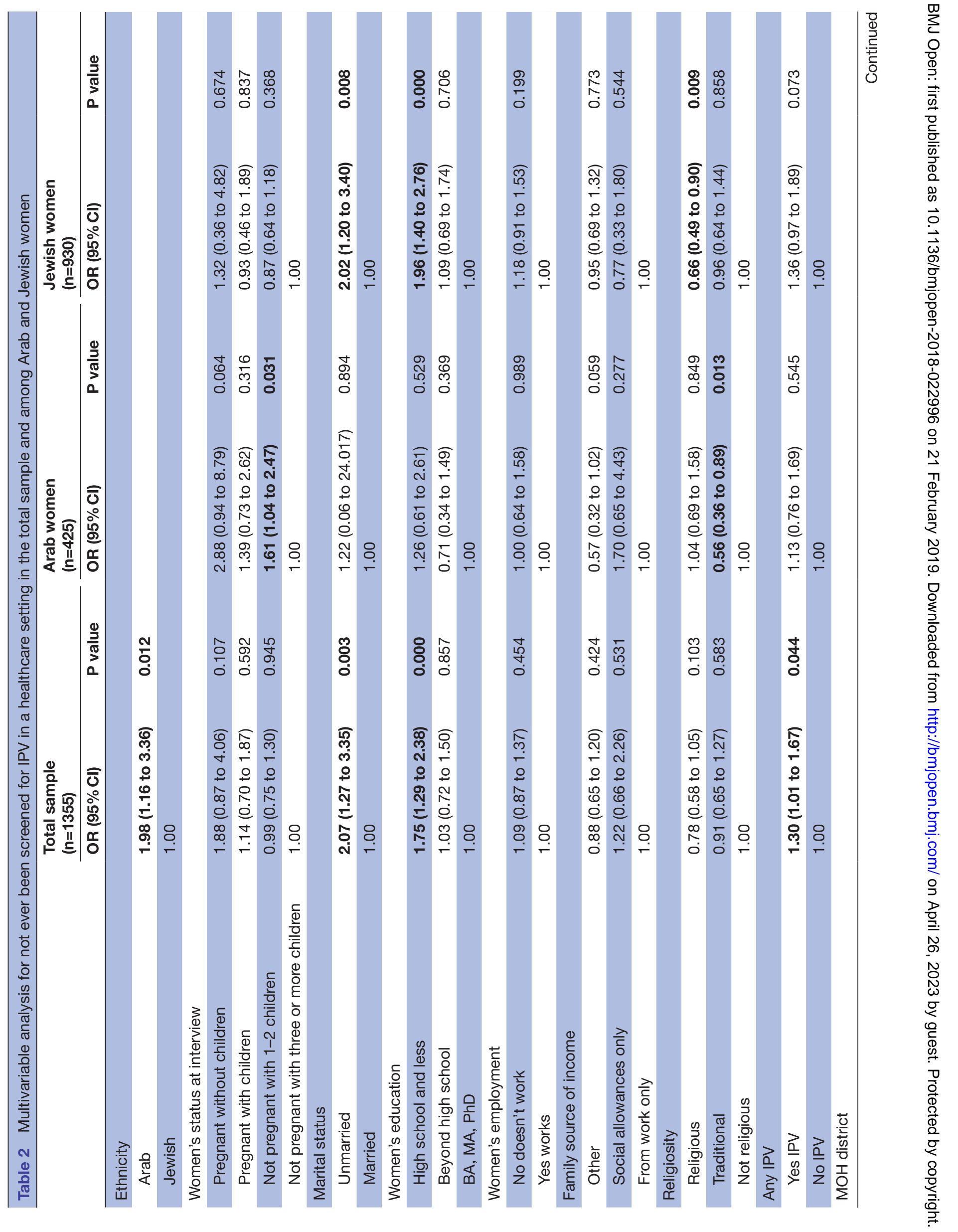




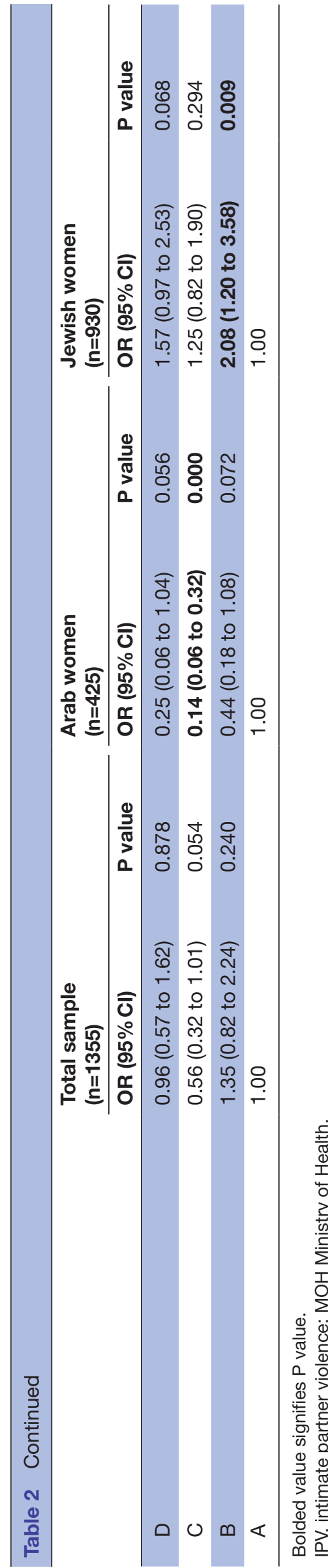

(OR, 95\% CI 1.57, 1.00 to 2.48). Arab women who did not report ES\&RI were almost three times more likely to be of older age, compared with younger age (OR,95\% CI 2.94, 1.04 to 8.30 ) and not pregnant compared with pregnant (OR,95\% CI $2.29,1.05$ to 4.99 and $2.04,1.16$ to 3.59 ). However, Arab women were less likely to report not having both ES\&RI if they live in district $\mathrm{D}$ and district C compared with district A (OR, 95\% CI 0.27, 0.10 to 0.72 and $0.35,0.15$ to 0.84 , respectively).

As for Jewish women, not reporting both ES\&RI was lower among women at older age compared with younger age. However, not reporting both ES \& RI was more likely among lower educated women compared with higher educated women (OR, 95\% CI 1.99, 1.34 to 2.95), and women living in district $\mathrm{D}$ and district $\mathrm{B}$ compared with district A (OR, 95\% CI 1.96, 1.29 to 2.97 and $1.95,1.20$ to 3.15 , respectively).

\section{DISCUSSION}

\section{IPV screening}

Screening for IPV is recommended within HCS in many countries, ${ }^{1627}$ as it may help women who experience IPV to disclose abuse and RI about supportive social and HCS. ${ }^{713}$ While there are different screening approaches, thus far, implementation of IPV screening across settings is incomplete. ${ }^{9}$ Insufficient system support for HCPs, as well as lack of skills and resources among HCPs are major barriers to fuller implementation. Therefore, healthcaresystem action is required to support these women. ${ }^{27}$ In the current study, we asked women of childbearing age if they had ES for IPV in Israel's HCS, and whether they had ever RI on what to do in case they experience IPV. We also created an index variable of both (ES\&RI). We found that despite the MOH Circular from 2003 on IPV, which mandates screening for every woman who interacts with HCS, including MCH clinics. this policy is not being followed consistently countrywide. Less than half (48.4\%) of our study participants reported ES for IPV via HCS. This result might be related to confusion among HCPs over screening methods, as the 2003 Circular is not conclusive in this regard. On the one hand, it mandates a universal screening, and on the other, it specifies conditions for case-finding. ${ }^{34}$ Despite this, our results are consistent with previous research on IPV screening. ${ }^{9}{ }^{12} \mathrm{~A}$ realist-informed systematic review of studies found that in most countries less than half of women were screened for IPV within $\mathrm{HCS} .{ }^{9}$ Based on our results it appears that HCPs in Israel need more health-system support to achieve greater screening coverage. Therefore, the $\mathrm{MOH}$ in Israel should offer consistent, sustained training to enhance knowledge among HCPs regarding IPV and IPV screening and remove barriers, including embarrassment, when screening women for IPV. ${ }^{35}$ Despite the fact that enhanced training for IPV screening might not increase the screening over mandatory screening, it can increase safety planning for women victims of IPV as was shown in the MOVE study in Australia. ${ }^{44}$ 
Table 3 Multivariable analysis for not receiving information about IPV services in a healthcare setting in the total sample and among Arab and Jewish women in Israel, 2014-2015

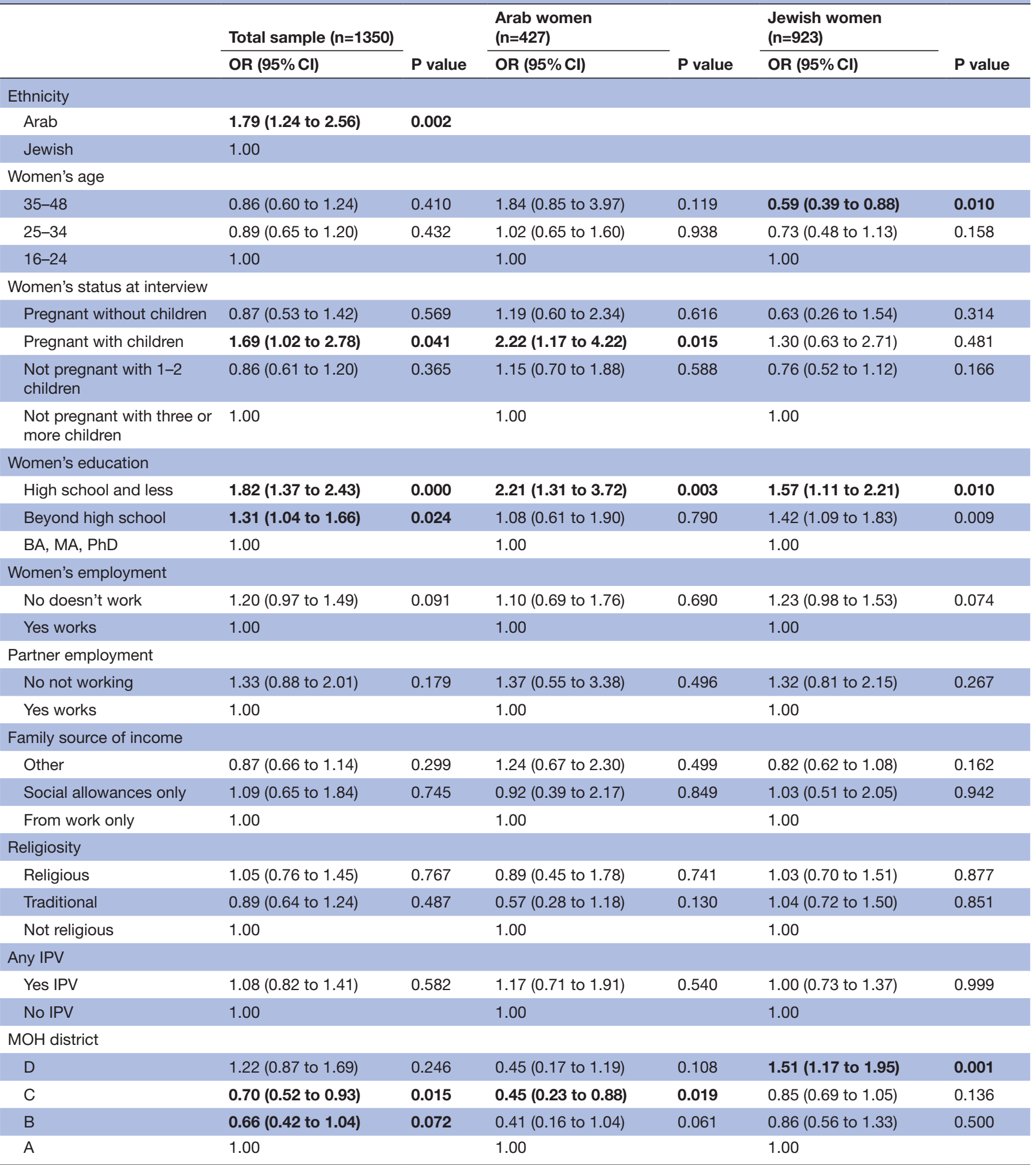

Bolded value signifies $P$ value.

IPV, intimate partner violence; $\mathrm{MOH}$, Ministry of Health.

Despite this, our finding that half the women in our sample were ES is encouraging, as previous studies in Israel have found far fewer reports of IPV screening. ${ }^{36} 45$
However, those studies included only small samples. One such study, conducted in the obstetrics and gynaecology department of a hospital in central Israel, found that only 
Table 4 Multivariable analysis for the combined variable of not ever being screened for IPV and not receiving information in a healthcare setting in the total sample and among Arab and Jewish women in Israel, 2014-2015

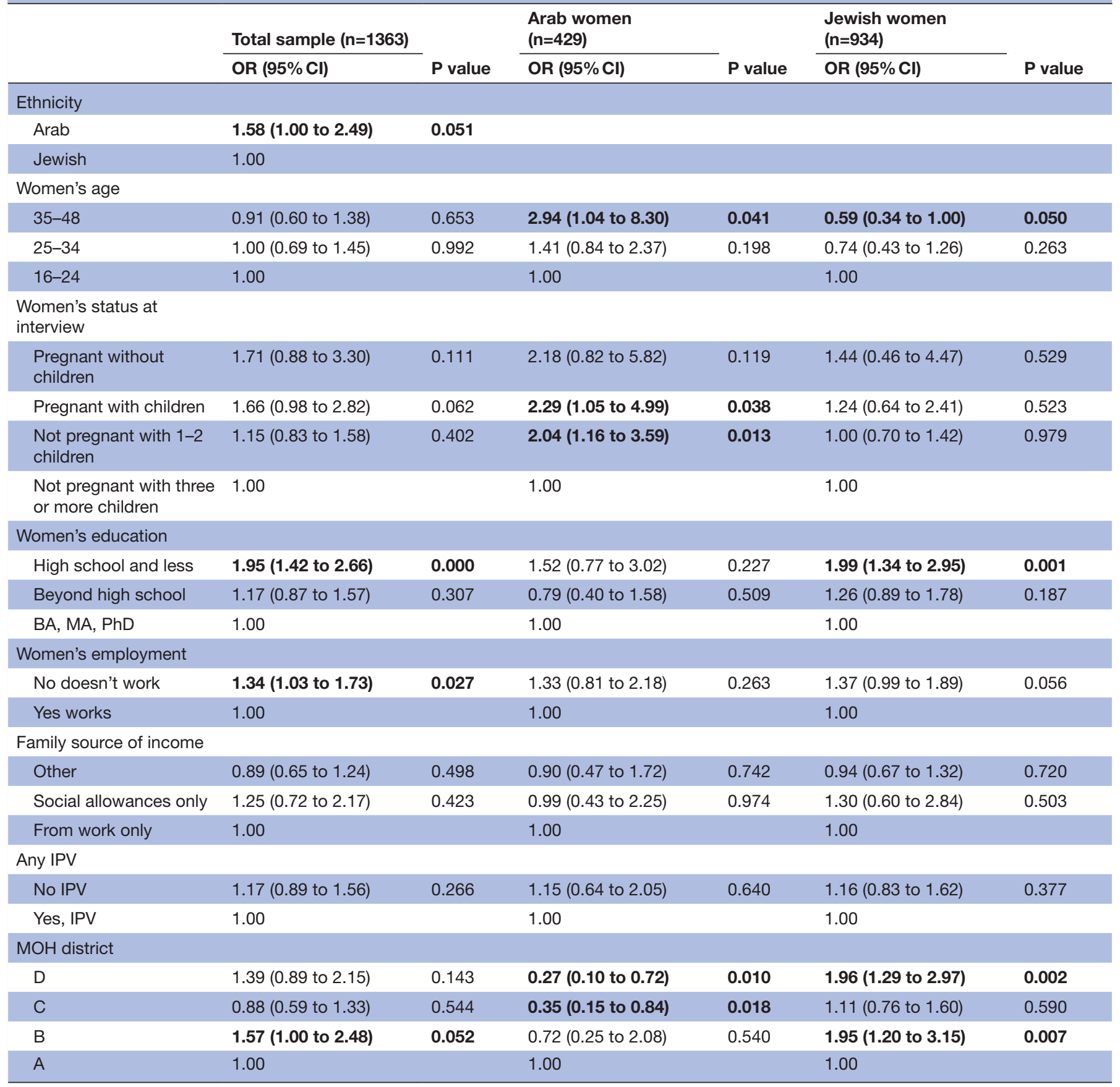

Bolded value signifies $P$ value.

IPV, intimate partner violence; $\mathrm{MOH}$ Ministry of Health.

$12 \%$ of women patients reported having been screened for domestic violence in the past year. ${ }^{36}$

\section{Associations between ES and IPV}

Another important finding from our study is that ES was not associated with most types of IPV variables. Only the association between ES and social and economic IPV was significant, but the direction of the association was opposite to our hypothesis: women exposed to social and economic IPV were less likely to ES. While these results might be related to power issues, due to smaller subsamples of women in our study who reported different types of IPV, it should raise the attention of policy-makers at the MOH.

The disparities we found in IPV screening based on women's ethnicity and socioeconomic status are of concern, as they show inequalities in screening between groups in Israel. Women who are at higher risk for IPV (Arab minority women, those with lower education and 
unmarried women) were less likely to be screened for IPV. Religiosity was also an important factor when we examined ES within each ethnic group. These findings also suggest that without a conclusive HCS screening protocol for IPV, some women at risk for IPV are not being identified. This is consistent with a qualitative study in the USA showing that a lack of a clear screening policy was a barrier to disclosure of IPV. ${ }^{25}$ This lack of a clear IPV screening policy might explain why ES was not significantly associated with any IPV and some specific IPV types in our study.

Our results about failure to screen women who are at risk of IPV might be related to different barriers, including a lack of training on their part for how to screen; lack of time in busy clinics; or reluctance to screen due to prejudices related to IPV. These barriers were mentioned in a MOH quality assessment report on IPV screening that was based on interviews with HCPs. ${ }^{35}$ While previous research shows that screening and referral alone are not sufficient to support women living with domestic violence, ${ }^{46}$ as these women have multiple social and health needs, ${ }^{47-49}$ enhanced training would help in increasing the safety planning for these women. ${ }^{44}$

\section{IPV screening by ethnicity}

The significant interaction we found between ethnicity and $\mathrm{MOH}$ district (area of living) in the association with ES, meanwhile, shows that IPV screening is implemented differentially among Arab and Jewish women in these districts. While we do not know the ethnic composition of HCPs, or whether Arab and Jewish women are reluctant to disclose IPV to HCPs of another or same ethnicity, previous research has shown that Arab women tend to use fewer professional services for coping with IPV, regardless of HCP ethnicity. ${ }^{43}$ Future research should examine HCP's attitudes, consider the prospect of cultural bias in IPV screening and examine whether HCPs make assumptions about the lack of support in the community for minority women who experience IPV. ${ }^{35}$

\section{Receiving information on supportive services}

Our results on RI regarding IPV services were similar to those on ES: just over half $(50.5 \%)$ of participants reported RI. However, any IPV, emotional IPV and social and economic IPV were associated with RI, but not in the direction we had hypothesised. Women who reported experiencing IPV consistently reported less RI. This association did not persist in the multivariate analysis, where, in the total sample of women, those with higher risk for IPV received less information. This included Arab women, pregnant women with children, women with lower (non-academic) education and those living in district A which is more economically disadvantaged compared with the other three districts. The multivariable analysis for Arab women, meanwhile, showed that women with children, not pregnant at the time of interview and women with less than high school education and living in district A (that has a higher concentration of Arab women living in economically disadvantaged conditions) were less likely to RI. Among Jewish women, younger (16-24years), lower educated women living in district D (more economically advantaged region compared with the other three) were less likely to RI.

Since, according to $\mathrm{MOH}$ policy in Israel, it is mandatory that all women RI about IPV services, provision of information should be reported by all study participants. However, we found that only about half of the women had RI on IPV services. RI should be recorded in all women's medical files, and our result can then be examined against those medical records. If the disparities are confirmed, this should be an alarm bell for policy-makers.

\section{ES and RI}

The combined variable we created that includes positive answers on ES\&RI confirmed our results for each of the variables when examined alone, but revealed an even poorer picture regarding IPV service implementation within HCS: only about one third of the women reported receiving both services. Further, the multivariable analysis for the combined variable showed that women at high risk for IPV were less likely to report receiving both services (ES\&RI). This included Arab, lower educated and unemployed women, as well as those living in district B (characterised by an ethnically mixed population). We do not know why these differences by district exist in our study, and we believe this requires future research. For Arab women, lower ES\&RI was associated with older age, not being pregnant at the time of interview and living in district A (economically disadvantaged area). Jewish women of younger ages (16-24years), with lower education and living in districts $\mathrm{D}$ (more economically advantaged) and B (characterised by ethnically mixed population) were less likely to have ES\&RI. Increased awareness among HCPs about the importance of providing both IPV services appears warranted, as well as institutional support for HCPs, including training and follow-up protocols to ensure documentation.

\section{Study limitations}

Our study makes a novel contribution to research assessing IPV screening within HCS. However, the study has some limitations that should be noted. First, our measure of IPV asks about any (current or past) events, but the timing of these acts, including when they began, is not known. Likewise, our interview asked about past screening, but did not specify when. Future research should ask when IPV began, as well as determine screening timing. Next, recall bias was likely in our study, as some women might not remember that they have been screened or RI. However, it is reasonable to assume that this is most likely to occur in women who had not experienced IPV, as it would often be of less importance to them. This could result in underestimation of the association between ES, RI or both and having experienced IPV. A selection bias might also be a possibility, as our study was based on a sample of women who visit MCH clinics of the MOH in Israel. Still, we used 
a stratified sample, and the sociodemographic characteristics of our sample are very close to those for women of reproductive age in Israel. ${ }^{50}$ Future research should examine screening among women in other HCS settings in Israel, such as primary care clinics, MCH clinics run by the Sick Funds (healthcare management organisations) and others in the municipalities of Jerusalem and Tel-Aviv. Our study sample size was sufficiently large to detect a difference of $10 \%$ in screening prevalence between women exposed and unexposed to IPV, assuming 50\% screening among unexposed women and $60 \%$ screening in exposed women, or $40 \%$ vs $50 \%$ (power $=95.3 \%$ ). We actually found a difference that was not statistically significant and in the opposite direction to our hypothesisthat is, that women unexposed to IPV were more likely to have been screened than exposed women $(49.6 \%$ vs $46.5 \%$ ). We believe it is safe to conclude that women exposed to IPV are not more likely to be screened than women not exposed to IPV. However, there might have been power issues for detecting the association between screening of IPV within each ethnic group (Arab and Jewish women), and specifically for Arab women (small sample). Future research should include larger samples of women from each of these ethnic groups in Israel.

\section{Conclusions and implications for policy and practice}

Despite Israel's long-standing IPV screening policy for all women visiting HCS, our study identified gaps in implementation of screening and information provision within HCS. Women at higher risk for IPV were less likely to have been screened or to have RI about IPV. These results call for further research to explore individual and systemlevel barriers to implementation of universal screening and provision of information on IPV among HCPs. Identifying and removing these barriers is vital, as our results show inequalities in conducting screening and information provision on support services, specifically for women at higher risk for IPV. HCPs in Israel need more healthsystem support to achieve greater screening coverage and to provide women with information on supportive services for IPV. Therefore, to aid in the early detection of IPV and prevent more severe IPV, the MOH in Israel should offer consistent, sustained training to enhance knowledge, change attitudes and remove barriers among HCPs regarding IPV screening and providing information about supportive services for IPV.

Acknowledgements We thank the women who agreed to participate in the study. Many thanks to the medical directors and head nurses of the Ministry of Health. We also thank the nurses at the $\mathrm{MCH}$ clinics who facilitated our data collection.

Contributors ND initiated the study, supervised all the study stages, conducted the data analysis and prepared the paper for publication. RS helped with data programming and data cleaning. ABP helped with data collection and prepared the study codebook. IS-V and PO'C contributed to the study design, critically read previous versions of the paper and suggested revisions. RL worked as a research assistant who helped in some updates of the background. All authors read and confirmed the final version of the paper.

Funding This work was supported by Israel Science Foundation (ISF) grant number $881 / 13$.
Competing interests None declared.

Patient consent for publication Not required.

Ethics approval The study was approved by the Human Subjects Research Committee of Ben-Gurion University of the Negev. Approval no. 1128-1.

Provenance and peer review Not commissioned; externally peer reviewed. Data sharing statement No additional data are available.

Open access This is an open access article distributed in accordance with the Creative Commons Attribution Non Commercial (CC BY-NC 4.0) license, which permits others to distribute, remix, adapt, build upon this work non-commercially, and license their derivative works on different terms, provided the original work is properly cited, appropriate credit is given, any changes made indicated, and the use is non-commercial. See: http://creativecommons.org/licenses/by-nc/4.0/.

\section{REFERENCES}

1. World Health Organization. Violence against women, fact sheet world health organization website: World Health Organization, 2016.

2. Dillon G, Hussain R, Loxton D, et al. Mental and physical health and intimate partner violence against women: a review of the literature. Int J Family Med 2013;2013:1-15.

3. Kruse M, Sørensen J, Brønnum-Hansen $\mathrm{H}$, et al. The health care costs of violence against women. $J$ Interpers Violence 2011;26:3494-508.

4. Bonomi $\mathrm{AE}$, Anderson ML, Rivara FP, et al. Health care utilization and costs associated with physical and nonphysical-only intimate partner violence. Health Serv Res 2009;44:1052-67.

5. Ansara DL, Hindin MJ. Formal and informal help-seeking associated with women's and men's experiences of intimate partner violence in Canada. Soc Sci Med 2010;70:1011-8.

6. Feder GS, Hutson M, Ramsay J, et al. Women exposed to intimate partner violence: expectations and experiences when they encounter health care professionals: a meta-analysis of qualitative studies. Arch Intern Med 2006;166:22-37.

7. Phelan MB. Screening for intimate partner violence in medical settings. Trauma Violence Abuse 2007;8:199-213.

8. O'Doherty LJ, Taft A, Hegarty K, et al. Screening women for intimate partner violence in healthcare settings: abridged Cochrane systematic review and meta-analysis. BMJ 2014;348:g2913.

9. O'Campo P, Kirst M, Tsamis C, et al. Implementing successful intimate partner violence screening programs in health care settings: evidence generated from a realist-informed systematic review. Soc Sci Med 2011;72:855-66.

10. Miller E, McCaw B, Humphreys BL, et al. Integrating intimate partner violence assessment and intervention into healthcare in the United States: a systems approach. J Womens Health 2015;24:92-9.

11. American Congress of Obstetricians and Gynecologists. ACOG Committee Opinion No. 518: Intimate partner violence. Obstet Gynecol 2012;119:412-7.

12. Sprague S, Slobogean GP, Spurr H, et al. A scoping review of intimate partner violence screening programs for health care professionals. PLoS One 2016;11:e0168502.

13. Nelson HD, Bougatsos C, Blazina I. Screening women for intimate partner violence: a systematic review to update the U.S. Preventive Services Task Force recommendation. Ann Intern Med 2012;156:796-808.

14. Wathen $\mathrm{CN}$, MacMillan $\mathrm{HL}$. Interventions for violence against women: scientific review. JAMA 2003;289:589-600.

15. WHO. Responding to intimate partner violence and sexual violence against women: WHO clinical and policy guidelines, 2013.

16. Wathen CN, Macgregor JC, Sibbald SL, et al. Exploring the uptake and framing of research evidence on universal screening for intimate partner violence against women: a knowledge translation case study. Health Res Policy Syst 2013;11:13.

17. Moyer VA. Screening for intimate partner violence and abuse of elderly and vulnerable adults: U.S. preventive services task force recommendation statement. Ann Intern Med 2013;158:478-86.

18. Lawoko S, Sanz S, Helström L, et al. Screening for Intimate Partner Violence against Women in Healthcare Sweden: Prevalence and Determinants. ISRN Nurs 2011;2011:1-7.

19. Feder G, Ramsay J, Dunne D, et al. How far does screening women for domestic (partner) violence in different health-care settings meet criteria for a screening programme? Systematic reviews of nine UK National Screening Committee criteria. Health Technol Assess 2009;13:37-47.

20. American Medical Association. Violence against women: relevance for medical practitioners. JAMA 1992;267:3184-9. 
21. American Medical Association. AMA data on violence between intimates:Intimate partner violence: 2000 interim meeting: american medical association web site. $2000 \mathrm{http}: / /$ www.ama-assn.org (Accessed 15th Sep 2011).

22. Sprague S, Madden K, Simunovic N, et al. Barriers to screening for intimate partner violence. Women Health 2012;52:587-605.

23. Gutmanis I, Beynon C, Tutty L, et al. Factors influencing identification of and response to intimate partner violence: a survey of physicians and nurses. BMC Public Health 2007;7:7-11.

24. Duncan MM, McIntosh PA, Stayton CD, et al. Individualized performance feedback to increase prenatal domestic violence screening. Matern Child Health J 2006;10:443-9.

25. Dichter ME, Wagner C, Goldberg EB, et al. Intimate partner violence detection and care in the veterans health administration: patient and provider perspectives. Womens Health Issues 2015;25:555-60.

26. Yonaka L, Yoder MK, Darrow JB, et al. Barriers to screening for domestic violence in the emergency department. $J$ Contin Educ Nurs 2007;38:37-45

27. García-Moreno C, Hegarty K, d'Oliveira AF, et al. The health-systems response to violence against women. The Lancet 2015;385:1567-79.

28. Zeitler MS, Paine AD, Breitbart V, et al. Attitudes about intimate partner violence screening among an ethnically diverse sample of young women. J Adolesc Health 2006;39:119.e1-119.e8.

29. Daoud N, Sergienko R, Shoham-Vardi I. Intimate partner violence prevalence, recurrence, types, and risk factors among arab, and jewish immigrant and nonimmigrant women of childbearing age in Israel. J Interpers Violence 2017:088626051770566.

30. Daoud N, Smylie J, Urquia M, et al. The contribution of socioeconomic position to the excesses of violence and intimate partner violence among aboriginal versus non-Aboriginal Women in Canada. Can J Public Health 2013;104:e278-83.

31. Costa D, Hatzidimitriadou E, loannidi-Kapolou E, et al. Male and female physical intimate partner violence and socio-economic position: a cross-sectional international multicentre study in Europe. Public Health 2016;139:44-52.

32. Ta Park VM, Hayes DK, Humphreys J. Disparities in intimate partner violence prenatal counseling: setting a baseline for the implementation of the guidelines for women's preventive services. Hawaii J Med Public Health 2014;73:137-43.

33. Daoud N, Sergienko R, Shoham-Vardi I. Intimate partner violence prevalence, recurrence, types, and risk factors among arab, and jewish immigrant and nonimmigrant women of childbearing age in Israel. J Interpers Violence 2017;63:088626051770566.

34. Lavi-Sahar Z. Laws and procedures on domestic violence: Jerusalem, Israel Ministry of Health, 2009.

35. Livni A, Zohar A, Meirovich A, et al. Quality assessment-Detection and treatment of victims of domestic violence in the community and in the hospital of the State of Israel (Hebrew: Jerusalem, Israel Ministry of Health, the Division of Quality Assurance, 2010.
36. Ben Natan M, Ben Ari G, Bader T, et al. Universal screening for domestic violence in a department of obstetrics and gynaecology: a patient and carer perspective. Int Nurs Rev 2012;59:108-14.

37. Gross R, Barmeli-Greenberg S. Health and welfare of women in Israel: findings of national survey Jerusalem: Israel Joint - Brookdale Institute [Hebrew], 2000.

38. WHO. Putting women first: ethical and safety recommendations for research on domestic violence against women: Department of Gender, Women and Health, WHO,, 2001.

39. Fisher M, Yassour-Borochowitz D, Neter E. Domestic abuse in pregnancy: results from a phone survey in northern Israel. Isr Med Assoc J 2003;5:35-9.

40. Slymen DJ, Hovell MF. Cluster versus individual randomization in adolescent tobacco and alcohol studies: illustrations for design decisions. Int J Epidemiol 1997;26:765-71.

41. US Preventive Services Task Force. Screening for family and intimate partner violence. Annals of internal medicine 2004;140:382-6.

42. Steinmetz S, Haj-Yahia MM. Definitions of and beliefs about wife abuse among ultra-Orthodox Jewish men from Israel. $J$ Interpers Violence 2006;21:525-54.

43. Haj-Yahia MM. Attitudes of arab women toward different patterns of coping with wife abuse. J Interpers Violence 2002;17:721-45.

44. Taft AJ, Hooker L, Humphreys C, et al. Maternal and child health nurse screening and care for mothers experiencing domestic violence (MOVE): a cluster randomised trial. BMC Med 2015;13:150.

45. Ben-Natan M. Screening by healthcare staff for domestic violence against women in israel - ethical and legal aspects. J Fam Violence 2014:29:809-13.

46. Reisenhofer S, Taft A. Women's journey to safety - the transtheoretical model in clinical practice when working with women experiencing intimate partner violence: a scientific review and clinical guidance. Patient Educ Couns 2013;93:536-48.

47. Daoud N, Matheson Fl, Pedersen C, et al. Pathways and trajectories linking housing instability and poor health among low-income women experiencing intimate partner violence (IPV): toward a conceptual framework. Women Health 2016;56:208-25.

48. O'Campo P, Daoud N, Hamilton-Wright S, et al. Conceptualizing housing instability: experiences with material and psychological instability among women living with partner violence. Housing Studies 2016;31:1-19.

49. Velonis AJ, Daoud N, Matheson F, et al. Strategizing safety: theoretical frameworks to understand women's decision making in the face of partner violence and social inequities. $J$ Interpers Violence 2017;32:3321-45

50. Central Bureau of Statistics (CBS). Live births, deaths and infant death, by district abd sub-district, population group and region Jerusalem: Central Bureau of Statistics (CBS), 2010. (Accessed May 2012). 